\title{
A principled method to identify individual differences and behavioral shifts in signaled active avoidance
}

\author{
Angelos-Miltiadis Krypotos, ${ }^{1}$ Justin M. Moscarello, ${ }^{2}$ Robert M. Sears, ${ }^{3,4,5}$ \\ Joseph E. LeDoux, ${ }^{3,4,6}$ and Isaac Galatzer-Levy ${ }^{7}$ \\ ${ }^{7}$ Department of Clinical Psychology, Utrecht University, Utrecht 3584 CS, The Netherlands; ${ }^{2}$ Department of Psychological and Brain \\ Sciences, Texas A\&M University, College Station, Texas 4235 TAMU, USA; ${ }^{3}$ Center for Neural Science, New York University, New York, \\ New York 10003, USA; ${ }^{4}$ Emotional Brain Institute, Nathan Kline Institute for Psychiatric Research, Orangeburg, New York 10962, USA; \\ ${ }^{5}$ Department of Child and Adolescent Psychiatry, New York University School of Medicine, New York, New York 10016, USA; \\ ${ }^{6}$ Department of Psychology, New York University, New York, New York 10003, USA; ${ }^{7}$ Department of Psychiatry, New York University \\ Langone Medical Center, New York, New York 10016, USA
}

Signaled active avoidance (SigAA) is the key experimental procedure for studying the acquisition of instrumental responses toward conditioned threat cues. Traditional analytic approaches (e.g., general linear model) often obfuscate important individual differences, although individual differences in learned responses characterize both animal and human learning data. However, individual differences models (e.g., latent growth curve modeling) typically require large samples and onerous computational methods. Here, we present an analytic methodology that enables the detection of individual differences in SigAA performance at a high accuracy, even when a single animal is included in the data set (i.e., $\mathrm{n}=1$ level). We further show an online software that enables the easy application of our method to any SigAA data set.

[Supplemental material is available for this article.]

Signaled active avoidance (SigAA) is the dominant experimental paradigm for studying instrumental defensive responses. During SigAA, an animal is typically placed in a shuttle box where it is exposed to pairings of a neutral stimulus (CS; e.g., a sound) with an aversive unconditioned stimulus (US; electric stimulation), with the animal being able to prevent the US by shuttling (i.e., crossing from one side of a divided chamber to the other) during the CS presentation (Mowrer and Lamoreaux 1942; Solomon and Wynne 1953). This simple procedure has been widely used across the fields of psychology and neuroscience for uncovering the theoretical principles and the neural underpinnings of (maladaptive) avoidance (LeDoux et al. 2017).

Statistical inferences of SigAA data are usually based on the analysis of average response rates, where a general linear model is fitted on the animals' mean responses (i.e., the traditional method). Despite the popularity of this approach, there are at least two limitations with it.

First, by averaging responses across the whole sample, the traditional method fails to account for individual differences in avoidance acquisition, with any deviations from the mean being essentially treated as noise (Lonsdorf and Merz 2017). However, there is good evidence that there is individual heterogeneity in the ability to acquire and extinguish instrumental responses to aversive stimuli (Galatzer-Levy et al. 2013; Galatzer-Levy 2014). Importantly, such heterogeneity has long been hypothesized to underlie fluctuations in psychological response to trauma (Bonanno et al. 2012).

Second, the traditional method fails to account for the phaseshift from a reactive threat detection state, where the animal acquires the CS-US associations (i.e., "Pavlovian learning") and freezes in presence of the CS, to an active state, where the animal

\section{Corresponding author: amkrypotos@gmail.com}

Article is online at http://www.learnmem.org/cgi/doi/10.1101/lm.047399.118. avoids the US presentation when the CS is presented (i.e., "instrumental learning"). Empirically, trajectories of individual differences characterized by these phase-shifts have been shown to better reflect avoidance acquisition than a linear pattern (Galatzer-Levy et al. 2014). This phase-shift (see above) is also predicted by the dominant theoretical accounts of avoidance learning (for reviews, see Krypotos et al. 2015; LeDoux et al. 2017).

Galatzer-Levy et al. (2014) showed that these shortcomings can be surpassed by using "latent class growth analysis" (LCGA), a cluster analytic method for identifying subgroups, within the sample data, defined by their rate of response change over time. LCGA refers to a range of statistical techniques that are used for estimating between individual differences, based on the data collected for each individual at multiple time points (i.e., repeated measures) (Curran et al. 2010). Specifically, LCGA uses repeated measurements per individual for classifying them into different groups, with each group showing heterogeneous response patterns from all other groups. Using LCGA, Galatzer-Levy et al. (2014) showed that the sample data could be reliably grouped into different subgroups, with each subgroup exhibiting a different rate of avoidance learning pattern (e.g., avoidance after one, two days etc.) or failing to learn to avoid within the allotted time. Importantly, the pattern of responses within the subgroups did not resemble the average group pattern.

The results above are important for at least two reasons. First, the LCGA results provided strong evidence of observable heterogeneity within the population as animals either did not learn to avoid at all, or acquired the instrumental response at different

\footnotetext{
C 2018 Krypotos et al. This article is distributed exclusively by Cold Spring Harbor Laboratory Press for the first 12 months after the full-issue publication date (see http://learnmem.cshlp.org/site/misc/terms.xhtml). After 12 months, it is available under a Creative Commons License (Attribution-NonCommercial 4.0 International), as described at http://creativecommons.org/licenses/by-nc/ $4.0 /$
} 
learning rates. Second, the subgroups that learned to avoid exhibited the predicted step-like pattern where, after acquiring the avoidance response, animals reached asymptotic levels of avoidance. As such, the theoretical prediction of a transition point from freezing to avoidance responses was supported.

Despite the advantages of LCGA over the traditional method for analyzing SigAA data, LCGA comes with onerous sample size requirements (Hertzog et al. 2006), diminishing their relevance to animal research which typically uses small samples to examine fine-grained mechanisms. In order to overcome these shortcomings, we have developed a principled method for categorizing animals in different trajectory learning clusters that is based on LCGA but has no high sample requirements. Our method is based on the definition of a predetermined inflection point after which animals exhibit asymptotic avoidance responses.

For determining this inflection point, we first reanalyzed the second study of Galatzer-Levy et al. (2014). Full details about the experimental setup and statistical analyses of this data set are described in the original study. In short, 186 naive male SpragueDawley rats performed a signaled active avoidance task as explained above, for $5 \mathrm{~d}$. The final LCGA solution suggested that the animals could be classified into four groups: Modal Avoiders ( $37 \%$ of the sample), Rapid Avoiders ( $22 \%$ of the sample), Slow Avoiders (22\% of the sample), and Non-Avoiders (20\% of the sample) (see Fig. 1).

As a further check of the LCGA classes, we followed up the results of Galatzer-Levy et al. (2014) by computing receiver operating characteristic (ROC) curves (Metz 1978). ROC is a graphical plot used to detect how well an algorithm is in classifying different individuals in two separate groups (Brown and Davis 2006). ROC curves are routinely used in, among others, self-report development for testing how accurately a scale can distinguish individuals with and without a disorder (e.g., depression). The most common measure for describing a ROC curve is by computing the area under it. Values between 0 and 0.60 indicate failure of the test to discriminate between a random case to a noncase whereas values closer to 1 indicate excellent discrimination. For these analyses, we compared each class defined by the LCGA, with the rest of the classes.

The results suggest that the group of animals were accurately placed into categories that can be adequately separated from each other (all ROC values $>0.80$; detailed values at Table 1 of the Supplemental Material and on the ROC plots of the Supple-

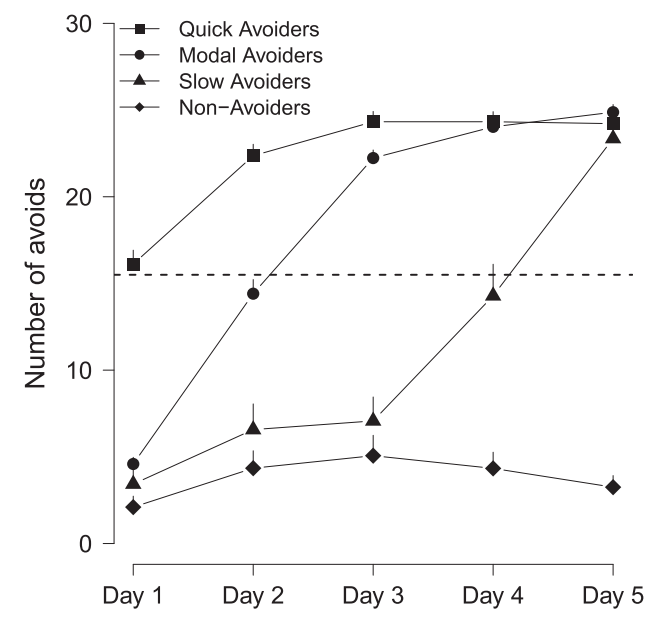

Figure 1. Visualization of the latent groups as identified by GalatzerLevy et al. (2014). Each point summarizes the mean responses for each group on a single day. Error bars denote standard errors. This figure is a reproduction of Figure 1 in Galatzer-Levy et al. (2014). mental Material). Based on these outcomes, we determined that the animals can be categorized into the different groups based on the rate of avoidance in any single day. Specifically, if the animal avoided the $55 \%$ of the trials on any single day (e.g., day 3 ) then this animal could be determined that has learned to avoid on that day, and be categorized accordingly (e.g., day 3 avoider). We followed up these analyses with also testing all inflation points from $5 \%$ to $100 \%$ (see inflation point plots in Supplemental Material). These results largely confirm the idea that $55 \%$ is a reliable point for categorizing animals in any of the categories. Specifically, by having this inflation point we ensured that the animals did not perform below chance levels, there were animals categorized on the first day (see Galatzer-Levy et al. 2014) and that there are at least two animals per category. ${ }^{8}$

Then, we tested the generalizability of our method by applying the predetermined inflection point (i.e., $55 \%$ of the trials being avoided on any single day) to a new independent, and unpublished, data set. Data set 2 was derived from a study with parameters similar to the experiments that produced data set 1 . As previously, the subjects of this study were male Sprague-Dawley rats. Training occurred over four daily sessions. The first trial of the first session was Pavlovian, in which a tone CS (15 sec, 70 $\mathrm{db})$ was followed by a shock US $(0.5 \mathrm{sec}, 0.7 \mathrm{~mA})$ regardless of the animal's behavior. On each subsequent trial, shuttling during the CS caused the US to be omitted and replaced with a blinking light confirmation signal. Each of the four daily sessions involved 30 of these avoidance trials. One important element of this study is that shuttling during the CS caused omission of the shock US, as opposed to termination of the CS and omission of the US, both of which occurred in the study that produced data set 1 . In addition, this study did not involve an escape contingency. Instead, the full $0.5 \mathrm{sec}$ duration of the US occurred on every trial in which the animal failed to perform the avoidance response (shuttling).

In Figure 2 we plot the results of each group for each day. The groups are defined based on when, if ever, was the first time they crossed the predetermined inflection point (55\%). In Figure 1 of the supplemental material we plot, for each day, the mean results of the animals who had crossed the predetermined inflection point (55\%), compared to the animals that had not. For investigating how well the predetermined inflection point (55\%) discriminated groups from each other, we carried a series of "default" Bayesian t-tests (Morey and Rouder 2015; Rouder et al. 2009) in which we compared, separate for each day, the mean avoidance of each group, to the mean distance between the mean responses of Avoiders and Non-Avoiders. We denote the relative support of the data under the alternative hypothesis, compared to the null hypothesis, by BF10.

The size of BF10 (detailed results in the Supplemental Table 2) suggests that there is a clear discrimination between classes. Specifically, apart from the Avoiders on the first day $(B F 10=$ $6.595)$, all BF10s provided strong (BF10 > 18.755) or unquestionable (BF10 > 100) support from the alternative hypothesis (i.e., differences between the mean responses of the group compared to the mean distance between the two groups) compared to the null hypothesis (i.e., no differences between the mean responses of the group compared to the mean distance between the two groups). Collectively, these results suggest that the predetermined inflection point (55\%) can be successfully applied to other data sets and meaningfully categorize groups of learners.

Lastly, we tested the split-half reliability of our method by fitting it to two random halves of a subset of data presented in

\footnotetext{
${ }^{8}$ Please note that although the $60 \%$ inflation point also fit these criteria, the differences between the $55 \%$ and the $60 \%$ inflation points is the categorization of just five animals.
} 


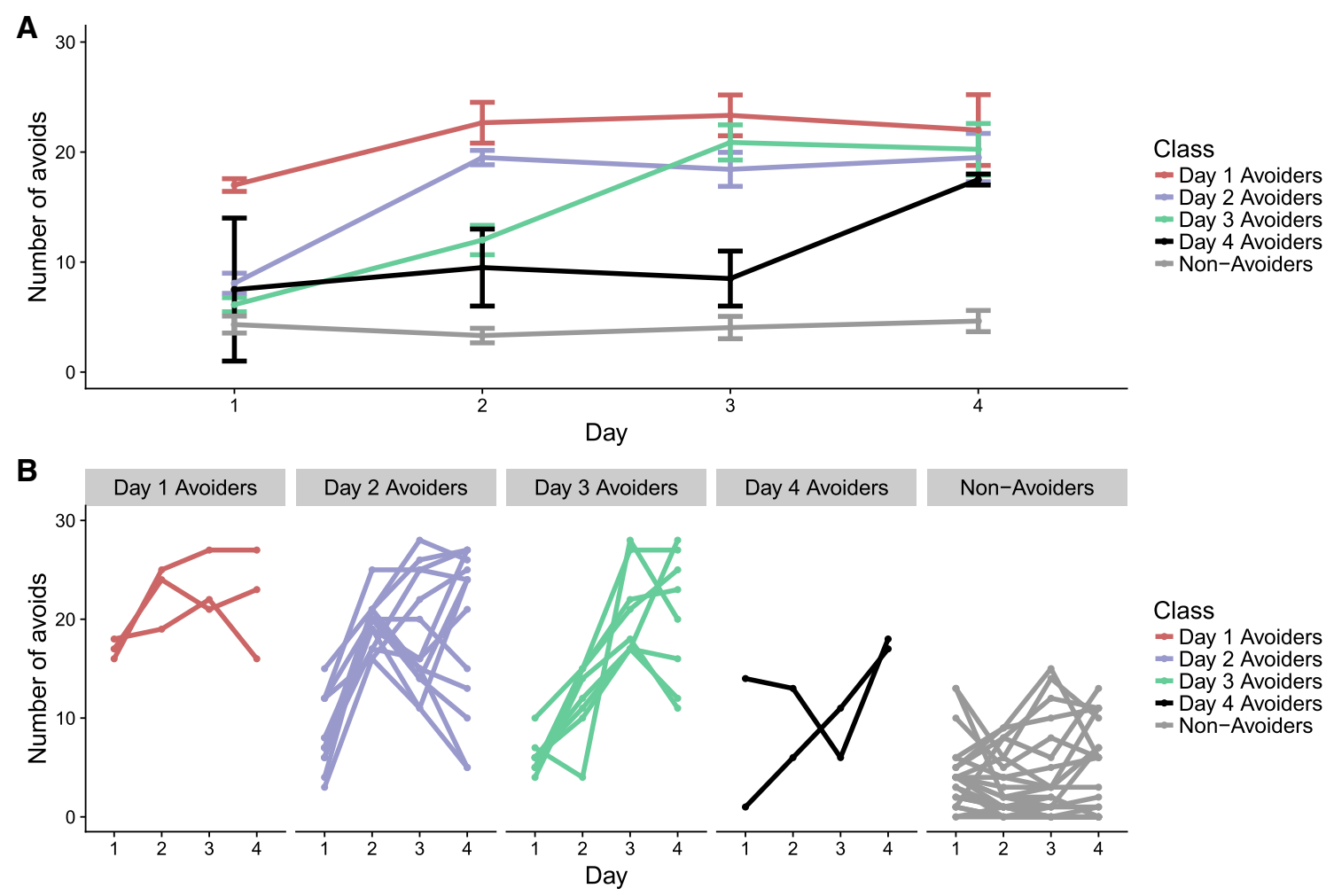

Figure 2. (A) Mean number of avoids for the second data set. Error bars denote standard errors. (B) Individual values for each subgroup, for the second data set.

Ramirez et al. (2015) (procedural details can be found in the original study). We present the data of 52 animals who undertook an avoidance learning procedure for $3 \mathrm{~d}^{9}$ In order to assess the reliability of our results, we split the data randomly into two equal data sets. We visualize the results of each data half on Figure 3 and in Supplemental Figure 2. On Supplemental Table 3 we present the Bayes factors for each half of the data set.

Collectively, the split-half results showed that the analyses of both halves resulted in similar outcomes. Specifically, the proposed predetermined inflection point (55\%) was able to classify rats as Avoiders and Non-Avoiders, with the Bayes factors providing good evidence for most of the groups, and the number of rats per group being largely similar. Collectively, these results show that the suggested predetermined inflection point (55\%) is reliable.

The presented method overcomes the key disadvantages of traditional analyses of SigAA. Specifically, by following our method, researchers are able to detect the expected learning heterogeneity within the tested sample, as well as the predicted step-wise pattern as suggested by individual differences analyses and most theoretical models of avoidance learning. As such, our method has many benefits of the LCGA for SigAA data but comes without requirements for large sample size in LCGA (Hertzog et al. 2006).

Our method can have significant impact on our understanding of mechanisms underlying SigAA as methods such as continuous recordings of neurons can now be utilized to understand

${ }^{9}$ Please note that after the third day, some of the rats were infused with muscimol or vehicle into the nucleus accumbens shell, or the nucleus accumbens core. The data collected during and after the treatment were not included in our data analyses and will not be described further. change from before to after phase shifts in behavior, and in the relevant brain regions of interest (e.g., with the method of optogenetics or chemogenetics). Further, manipulations can be utilized to determine if they cause a shift between active avoidance and the reactive threat detection state. Both of these approaches are relevant for understanding mechanisms of health and pathology in response to environmental stress, but are not accessible when examining a linear trajectory of change over time.

To make our methodology readily available to every researcher, we have created a user friendly and free software that is available at https://utrecht-university.shinyapps.io/aacc/. Given its intuitive design, every researcher can use our application easily with the relevant outcomes being available in seconds. The wide application of our methodology to SigAA data sets could lead to significant theoretical and clinically relevant advancements.

Here, we presented our approach using conditioning avoidance data. However, our approach could be extended for other data sets, even across species. For example, it could be tested whether such a predetermined inflection point (55\%) could be detected also in human avoidance data set (e.g., in Vervliet and Indekeu 2015). Given the recent interest in avoidance learning in humans, answering such questions seems timely. In addition, it would be easy to see our method extended to other responses (e.g., skin conductance or the startle reflex), such as for detecting when learning has been achieved during a Pavlovian threat acquisition phase.

Our data-driven method comes to be added to other statistical methods for detecting differences in learning (e.g., Bush et al. 2007; Reznikov et al. 2015; King et al. 2017; Shumake et al. 2018). Although an one-to-one comparison among the different approaches is beyond the scope of this paper, two characteristics that differentiate our method from previous methods is that 

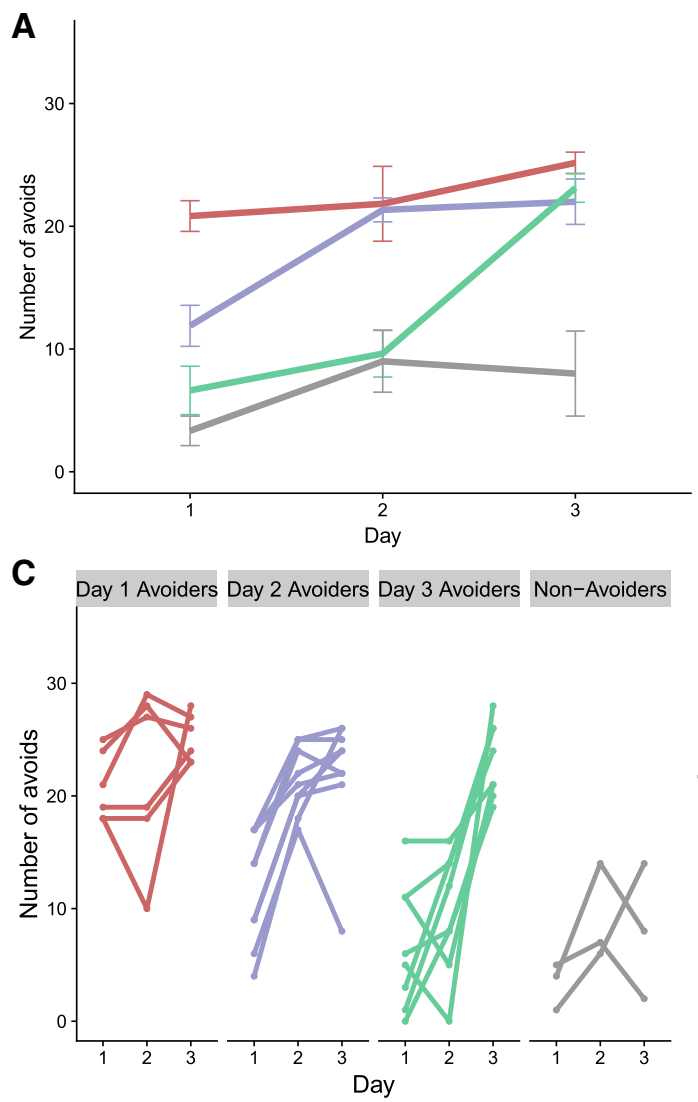

B
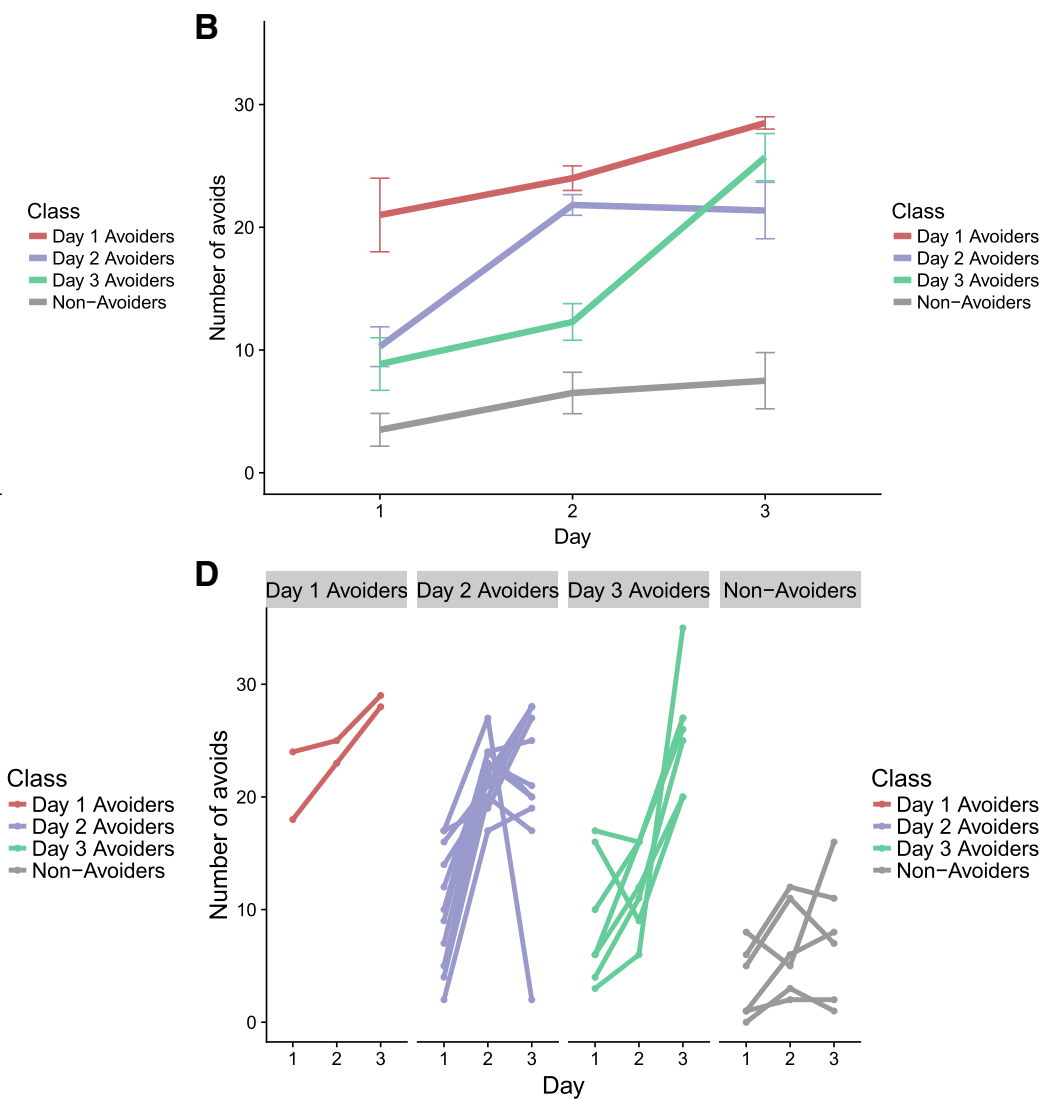

Figure 3. (A) Mean number of avoids for each subgroup, for the first half of the third data set. Error bars denote standard errors. (B) Mean number of avoids for each subgroup, for the second half of the third data set. Error bars denote standard errors. (C) Individual values for each subgroup, for the first half of the third data set. $(D)$ Individual values for each subgroup, for the second half of the third data set.

(a) we refer to avoidance learning data, something that previous studies have not addressed adequately, and (b) that our method can be used even with small samples. In any case, we believe that our method comes handy in the arsenal of methods that researchers can use for addressing individual differences in learning. We believe that more studies in this area of research is timely.

Despite the advantages of our method over the traditional approach, we acknowledge three potential issues. First, in case of large samples, we would urge researchers to still take advantage of the flexibility of the LCGA analyses. Second, it could be argued that any use of a cutoff (here the predetermined inflection point, $55 \%$ ) is debatable, especially when participants perform close to the cutoff scores. Third, our method considers only sum of avoidance responses per each training day. At the same time, given the advantages of our method (see above) we do believe that there is little reason not to use our methodology when small sample sizes are used, with larger studies further testing and validating the initial conclusions.

In sum, we have presented a principled way, with a userfriendly software, to classify animals undergoing a SigAA into distinct subpopulations that overcomes many of the limitations of the traditional analytic methods. As we have argued above, the wide application of our methodology to SigAA could lead to significant new theoretical and mechanistic insights.

\section{Acknowledgments}

Part of the reported research was supported by a grant from Neuroscience and Cognition Utrecht.

\section{References}

Bonanno GA, Kennedy P, Galatzer-Levy IR, Lude P, Elfström ML. 2012. Trajectories of resilience, depression, and anxiety following spinal cord injury. Rehabil Psychol 57: 236.

Brown CD, Davis HT. 2006. Receiver operating characteristics curves and related decision measures: a tutorial. Chemometr Intell Lab Syst 80: 24-38.

Bush DE, Sotres-Bayon F, LeDoux JE. 2007. Individual differences in fear: isolating fear reactivity and fear recovery phenotypes. J Trauma Stress 20: 413-422.

Curran PJ, Obeidat K, Losardo D. 2010. Twelve frequently asked questions about growth curve modeling. J Cogn Dev 11: 121-136.

Galatzer-Levy IR. 2014. Empirical characterization of heterogeneous posttraumatic stress responses is necessary to improve the science of posttraumatic stress. J Clin Psychiatry 75: e950-e952.

Galatzer-Levy IR, Ankri Y, Freedman S, Israeli-Shalev Y, Roitman P, Gilad M, Shalev AY. 2013. Early PTSD symptom trajectories: persistence, recovery, and response to treatment: results from the Jerusalem Trauma Outreach and Prevention Study (J-TOPS). PLoS One 8: e70084.

Galatzer-Levy IR, Moscarello J, Blessing EM, Klein J, Cain CK, LeDoux JE. 2014. Heterogeneity in signaled active avoidance learning: substantive and methodological relevance of diversity in instrumental defensive responses to threat cues. Front Syst Neurosci 8: 179

Hertzog C, Lindenberger U, Ghisletta P, Oertzen Tv. 2006. On the power of multivariate latent growth curve models to detect correlated change. Psychol Methods 11: 244-252.

King G, Scott E, Graham BM, Richardson R. 2017. Individual differences in fear extinction and anxiety-like behavior. Learn Mem 24: 182-190.

Krypotos AM, Effting M, Kindt M, Beckers T. 2015. Avoidance learning: a review of theoretical models and recent developments. Front Behav Neurosci 9: 189.

LeDoux J, Moscarello J, Sears R, Campese V. 2017. The birth, death and resurrection of avoidance: a reconceptualization of a troubled paradigm. Mol Psychiatry 22: 24-36. 
Lonsdorf TB, Merz CJ. 2017. More than just noise: inter-individual differences in fear acquisition, extinction and return of fear in humansbiological, experiential, temperamental factors, and methodological pitfalls. Neurosci Biobehav Rev 80: 703-728.

Metz CE. 1978. Basic principles of ROC analysis. Semin Nucl Med 8: 283-298.

Morey RD, Rouder JN. 2015. BayesFactor: computation of Bayes factors for common designs [Computer software manual]. Retrieved from https://CRAN.R-project.org/package=BayesFactor $(\mathrm{R}$ package version $0.9 .12-2)$

Mowrer OH, Lamoreaux RR. 1942. Avoidance conditioning and signal duration-a study of secondary motivation and reward. Psychol Monogr 54: $1-34$.

Ramirez F, Moscarello JM, LeDoux JE, Sears RM. 2015. Active avoidance requires a serial basal amygdala to nucleus accumbens shell circuit. J Neurosci 35: 3470-3477.

Reznikov R, Diwan M, Nobrega JN, Hamani C. 2015. Towards a better preclinical model of PTSD: characterizing animals with weak extinction, maladaptive stress responses and low plasma corticosterone. J Psychiatr Res 61: 158-165.

Rouder JN, Speckman PL, Su D, Morey RD, Iverson G. 2009. Bayesian $t$ tests for accepting and rejecting the null hypothesis. Psychon Bull Rev 16: $225-237$.

Shumake J, Jones C, Auchter A, Monfils MH. 2018. Data-driven criteria to assess fear remission and phenotypic variability of extinction in rats. Philos Trans R Soc Lond B Biol Sci 373: 20170035.

Solomon RL, Wynne LC. 1953. Traumatic avoidance learning: acquisition in normal dogs. Psychol Monogr Gen Appl 67: 1-19.

Vervliet B, Indekeu E. 2015. Low-cost avoidance behaviors are resistant to fear extinction in humans. Front Behav Neurosci 9: 351.

Received February 2, 2018; accepted in revised form August 7, 2018. 


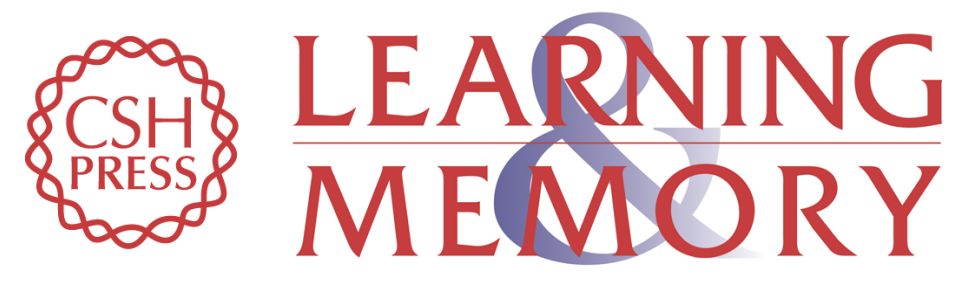

\section{A principled method to identify individual differences and behavioral shifts in signaled active avoidance}

Angelos-Miltiadis Krypotos, Justin M. Moscarello, Robert M. Sears, et al.

Learn. Mem. 2018, 25:

Access the most recent version at doi:10.1101/Im.047399.118

\section{Supplemental http://learnmem.cshlp.org/content/suppl/2018/10/09/25.11.564.DC1 Material}

References This article cites 20 articles, 2 of which can be accessed free at: http://learnmem.cshlp.org/content/25/11/564.full.html\#ref-list-1

Creative This article is distributed exclusively by Cold Spring Harbor Laboratory Press for the Commons first 12 months after the full-issue publication date (see

License http://learnmem.cshlp.org/site/misc/terms.xhtml). After 12 months, it is available under a Creative Commons License (Attribution-NonCommercial 4.0 International), as described at http://creativecommons.org/licenses/by-nc/4.0/.

Email Alerting Receive free email alerts when new articles cite this article - sign up in the box at the Service top right corner of the article or click here. 